\title{
ON THE INDEPENDENCE OF UNDEFINED IDEAS
}

\author{
BY J. C. C. MCKINSEY
}

Considerable attention has been devoted recently to the independence of postulates, but little notice has been paid to the question of the independence of the undefined ideas occurring in postulate sets. It is true that Padoa* has given a definition of independence of undefined ideas and has constructed a test to show its presence; but, during the thirty-four years which have followed his work, no one, so far as I know, has discussed the concept or applied the test. I therefore feel that it may not be out of place again to define this concept and to attempt to justify the test for it. I also undertake to exhibit the importance of the independence of undefined ideas by showing the situation which arises when it is lacking.

Suppose $S$ is an abstract mathematical system with undefined ideas $\left(K_{1}, K_{2}, \cdots ; R_{1}, R_{2}, \cdots ; \odot_{1}, \odot_{2}, \cdots\right)$, where $K_{i}$ represents an undefined class, $R_{i}$ an undefined relation, and $\odot_{i}$ an undefined operation. Then we say that any one of the classes, as $K_{1}$, is dependent on the other undefined ideas if there exists in $S$ a theorem such as the following:

$$
\left(x \in K_{1}\right) \equiv F\left(x ; K_{2}, \cdots ; R_{1}, R_{2}, \cdots ; \odot_{1}, \odot_{2}, \cdots\right),
$$

where the triple bar indicates mutual implication. Similarly we say that $R_{1}$ is dependent if there exists in $S$ a theorem such as

$$
\left(x R_{1} y\right) \equiv G\left(x, y ; K_{1}, K_{2}, \cdots ; R_{2}, \cdots ; \odot_{1}, \odot_{2}, \cdots\right),
$$

and that $\odot_{1}$ is dependent if there exists in $S$ a theorem such as

$$
\left(x \odot_{1} y\right)=H\left(x, y ; K_{1}, K_{2}, \cdots ; R_{1}, R_{2}, \cdots ; \odot_{2}, \cdots\right),
$$

where the double bar indicates identity. (It is important to notice that, in (1), the only undefined symbol occurring on the left is $K_{1}$, and that $K_{1}$ does not occur on the right; similarly for $R_{1}$ and $\odot_{1}$, respectively, in (2) and (3)). If an undefined idea is not dependent, it is said to be independent.

* Essai d'une théorie algébrique des nombres entiers, prêcédé d'une introduction logique à une théorie déductive quelconque, Bibliothéque du Congrès International de Philosophie, vol. 3 (1900). 
The importance of the independence of undefined ideas lies in the fact that if notions are not independent it is possible to define one of them in terms of the others without changing the character of $S$. And such a definition will, in general, make it possible to reduce the number of postulates required for $S$. This is true because, so far as deductive power is concerned, it makes no difference whether a proposition is given as a postulate or as a definition.

The following example will perhaps make this clearer: Huntington* has given a complete set of postulates for the algebra of complex quantities, in which he takes as undefined the five ideas $(K, C, \oplus, \odot, \ominus)$, where $K$ is the class of complex quantities, and $C$ the sub-class of real quantities. From the postulates given it is possible to deduce the following theorem.

$A$ necessary and sufficient condition that $a \ominus b$ is that the following three propositions be true:

1. $a$ and $b$ belong to $C$,

2. $a \neq b$,

3. there exists a $t$, belonging to $C$, such that $a \oplus(t \odot t)=b$.

Since this theorem holds in the system, it is evident that we can define the relation $\theta$ as follows:

$$
a \ominus b:=::(a, b \in C)::(a \neq b)::(\exists t):(t \in C) \cdot(a \oplus(t \odot t)=b) \text {. Def. }
$$

For this definition would not allow the deduction of anything which would not follow, anyhow, from the theorem. Also, although Huntington's postulates, without the definition, are independent, they become dependent in the presence of the definition. For P15 (which asserts that if $a \ominus b$, then $a$ and $b$ are distinct) is given directly in the definition. And it is easy to deduce the following postulates from other postulates in the presence of the definition.

P18. If $x \ominus y$, then $a \oplus x \ominus a \oplus y$.

P19. If $z \ominus a$ and $z \ominus b$, where $z$ is the zero element, then $z \ominus a \odot b$.

If the ideas are independent, on the other hand, then to define one of them in terms of the others is to change the character of $S$ from its character as given by the postulates. This does not mean, it should be noticed, that it is not possible to give a

* Monographs on Topics of Modern Mathematics, pp. 186-190, or Transactions of this Society, vol. 6 (1905), pp. 209-229. 
definition in such a case, but merely that it is impossible to give a definition without introducing new information. Thus suppose, for example, that our system* involves only the two undefined ideas $K$ and $\oplus$ and the single postulate: $K$ consists of just two elements, 0 and 1 . Then it is seen that $\oplus$ is independent of $K$ in the sense defined above, but clearly we may define $\oplus$ in any one of sixteen ways by means of a table

\begin{tabular}{l|l|l|}
$\oplus$ & 0 & $\frac{1}{0}$ \\
\hline 1 & $\frac{l_{1}}{t_{3}}$ & $\frac{l_{2}}{l_{4}}$ \\
\hline
\end{tabular}

$$
\left(l_{i}=0 \text { or } 1\right) \text {. }
$$

But it is evident that making any one of the possible definitions will change the nature of the system, since any definition will enable us to prove propositions which could not be proved from the postulate alone. If the primitive ideas of a categorical system are independent, however, then any definition of one of them in terms of the others will make the system inconsistent.

I now set forth a method whereby, if $K_{1}$ is independent of $\left(K_{2}, \cdots ; R_{1}, R_{2}, \cdots ; \odot_{1}, \odot_{2}, \cdots\right)$, this independence may be shown. Let $\bar{S}$ be a concrete representation of $S$, so that $K_{i}$ is interpreted concretely as $\bar{K}_{i}, R_{i}$ as $\bar{R}_{i}$, and $\odot_{i}$ as $\bar{\odot}_{i}$. Let $\bar{S}$ be a second concrete interpretation of $S$, so that $K_{1}$ is interpreted as $\bar{K}_{1}, K_{i}$ as $\bar{K}_{i}$ for $i \neq 1, R_{i}$ as $\bar{R}_{i}$, and $\odot i$ as $\bar{\odot}_{i}$. Let $\bar{K}_{i}$ and $\bar{K}_{1}$ differ in their extension, so that there is an element $a$ which belongs to $\bar{K}_{1}$ but not to $\bar{K}_{1}$. If it is possible to find $\bar{S}$ and $\bar{S}$ as specified, then $K_{1}$ is independent of $\left(K_{2}, \cdots ; R_{1}, R_{2}, \cdots\right.$; $\left.\odot_{1}, \odot_{2}, \cdots\right)$. For if $K_{1}$ were dependent, then we should have

$$
\left(x \in K_{1}\right) \equiv F\left(x ; K_{2}, \cdots ; R_{1}, R_{2}, \cdots ; \odot_{1}, \odot_{2}, \cdots\right) .
$$

Hence, in $\bar{S}$,

$$
\left(x \in \bar{K}_{1}\right) \equiv F\left(x ; \bar{K}_{2}, \cdots ; \bar{R}_{1}, \bar{R}_{2}, \cdots ; \bar{\odot}_{1}, \bar{\odot}_{2}, \cdots\right) .
$$

And in $\bar{S}$,

$$
\left(x \in \bar{K}_{1}\right) \equiv F\left(x ; \bar{K}_{2}, \cdots ; \bar{R}_{1}, \bar{R}_{2}, \cdots ; \bar{\odot}_{1}, \bar{\bigodot}_{2}, \cdots\right) .
$$

Thus, since $F$ is single-valued, and since material equivalence is transitive,

* I am indebted to B. A. Bernstein for this example, and for several other suggestions. 


$$
x \epsilon \bar{K}_{1} \equiv x \epsilon \bar{K}_{1} .
$$

Hence, in particular,

$$
a \epsilon \bar{K}_{1} \equiv a \epsilon \bar{K}_{1} .
$$

But by hypothesis $a \epsilon K_{1}$ is true and $a \epsilon \bar{K}_{1}$ is false, so that (8) is absurd. Hence, if the systems $\bar{S}$ and $\bar{S}$ can be found, $K_{1}$ is independent.

In a similar way, if we can find two interpretations $\left(\bar{K}_{1}, \bar{K}_{2}, \cdots ; \bar{R}_{1}, \bar{R}_{2}, \cdots ; \bar{\odot}_{1}, \bar{\odot}_{2}, \cdots\right)$ and $\left(\bar{K}_{1}, \bar{K}_{2}, \cdots\right.$; $\left.\bar{R}_{1}, \bar{R}_{2}, \cdots ; \bar{\odot}_{1}, \bar{\odot}_{2}, \cdots\right)$, then an argument like that used above will show that $R_{1}$ is independent; and similarly for the operations. It is important to notice, however, that in order that $\bar{R}_{1}$ be different from $\bar{R}_{1}$, it must differ in extension, that is, there must be an ordered couple $(a, b)$ such that $a \bar{R}_{1} b$ is true and $a \bar{R}_{1} b$ is false. And in order that $\bar{\odot}_{1}$, differ from $\bar{\odot}_{1}$ there must be an ordered triple $(a, b, c)$ such that*

$$
a \bar{\odot}_{1} b=c, \quad \text { and } \quad a \bar{\odot}_{1} b \neq c .
$$

I now illustrate the application of the method by an example. For this purpose I make use of the following system $S$, due to Huntington. $\dagger$

Undefined ideas:

$K=$ an undefined class of elements, $a, b, c, \cdots$.

$C=$ an undefined subclass within $K$.

$a+b=$ the result of an undefined binary operation on $a$ and $b$.

$a^{\prime}=$ the result of an undefined unary operation on $a$.

It is assumed that $C$, and hence $K$, is non-empty.

Postulates.

P1. If $a$ is in $K$ and $b$ is in $K$, then $a+b$ is in $K$.

P2. If $a$ is in $K$, then $a^{\prime}$ is in $K$.

P3. If $a$ is in $C$, then $a$ is in $K$.

* Padoa's failure to indicate any criterion for the "differentness" of two interpretations is perhaps more than anything else responsible for the reluctance of mathematicians to employ his test.

$\dagger$ Independent postulates for the informal part of Principia Mathematica, this Bulletin, vol. 40 (1934), pp. 127-136. 
P4. If $a+b$ is in $C$, then $b+a$ is in $C$.

P5. If $a$ is in $C$, then $a+b$ is in $C$.

Definition. The notation ( $a$ is in $C^{\prime}$ ) shall mean ( $a$ is in $K$ and $a$ is not in $C$ ).

P6. If $a$ is in $K$ and $a^{\prime}$ is in $C$, then $a$ is in $C^{\prime}$.

P7. If $a$ is in $K$ and $a^{\prime}$ is in $C^{\prime}$, then $a$ is in $C$.

P8. If $a+b$ is in $C$ and $a^{\prime}$ is in $C$, then $b$ is in $C$.

I now show that the four ideas $\left(t,{ }^{\prime}, C, K\right)$ are independent.

\begin{tabular}{|c|c|c|c|c|c|c|c|}
\hline \multirow{5}{*}{$S_{1.1}$} & \multirow{5}{*}{$\begin{aligned} K & =1,2,3 \\
C & =1,2\end{aligned}$} & \multicolumn{4}{|c|}{ INDEPENDENCE OF $†$} & \multirow[b]{2}{*}{$a$} & \multirow[b]{2}{*}{$a^{\prime}$} \\
\hline & & + & 1 & 2 & 3 & & \\
\hline & & 1 & 1 & 1 & 1 & 1 & 3 \\
\hline & & 2 & 1 & 2 & 2 & 2 & 3 \\
\hline & & 3 & 1 & 2 & 3 & 3 & 1 \\
\hline \multirow{4}{*}{$S_{1.2}$} & \multirow{4}{*}{$\begin{aligned} K & =1,2,3 \\
C & =1,2\end{aligned}$} & + & 1 & 2 & 3 & $a$ & $a^{\prime}$ \\
\hline & & 1 & 1 & 1 & 1 & 1 & 3 \\
\hline & & 2 & 1 & 1 & 1 & 2 & 3 \\
\hline & & 3 & 1 & 1 & 3 & 3 & 1 \\
\hline
\end{tabular}

Thus it is seen that there are two systems $S_{1.1}$ and $S_{1.2}$, each of which satisfies P1-P8 and in each of which $K, C$, and ' have the same specification, while + is specified differently in $S_{1.1}$ and $S_{1.2}$. Hence + cannot be defined in terms of $K, C$, and ' without changing the character of $S$.

\begin{tabular}{|c|c|c|c|c|c|c|c|}
\hline \multirow{5}{*}{$S_{2.1}$} & \multicolumn{7}{|c|}{ INDEPENDENCE OF ${ }^{\prime}$} \\
\hline & & + & 1 & 2 & 3 & $a$ & $a^{\prime}$ \\
\hline & $\Lambda=1,2,0$ & 1 & 1 & 1 & 1 & 1 & 3 \\
\hline & & 2 & 1 & 2 & 2 & 2 & 3 \\
\hline & & 3 & 1 & 2 & 3 & 3 & 1 \\
\hline \multirow{4}{*}{$S_{2.2}$} & \multirow{4}{*}{$\begin{aligned} K & =1,2,3 \\
C & =1,2\end{aligned}$} & + & 1 & 2 & 3 & $a$ & $a^{\prime}$ \\
\hline & & 1 & 1 & 1 & 1 & 1 & 3 \\
\hline & & 2 & 1 & 2 & 2 & 2 & 3 \\
\hline & & 3 & 1 & 2 & 3 & 3 & 2 \\
\hline
\end{tabular}




\section{INDEPENDENCE* OF $C$}

\begin{tabular}{|c|c|c|c|c|c|c|c|c|}
\hline \multirow{5}{*}{$S_{3.1}$} & \multirow{5}{*}{$\begin{aligned} K & =1,2,3,4 \\
C & =1,2\end{aligned}$} & + & 1 & 2 & 3 & 4 & $a$ & $a^{\prime}$ \\
\hline & & 1 & 1 & 1 & 1 & 1 & 1 & 4 \\
\hline & & 2 & 1 & 2 & 1 & 2 & 2 & 3 \\
\hline & & 3 & 1 & 1 & 3 & 3 & 3 & 2 \\
\hline & & 4 & 1 & 2 & 3 & 4 & 4 & 1 \\
\hline \multirow{5}{*}{$S_{3.2}$} & \multirow{5}{*}{$\begin{aligned} K & =1,2,3,4 \\
C & =1,3\end{aligned}$} & + & 1 & 2 & 3 & 4 & $a$ & $a^{\prime}$ \\
\hline & & 1 & 1 & 1 & 1 & 1 & 1 & 4 \\
\hline & & 2 & 1 & 2 & 1 & 2 & 2 & 3 \\
\hline & & 3 & 1 & 1 & 3 & 3 & 3 & 2 \\
\hline & & 4 & 1 & 2 & 3 & 4 & 4 & 1 \\
\hline
\end{tabular}

INDEPENDENCE OF $K$

$\begin{aligned} S_{4.1} \quad K & =1,2 \\ C & =1\end{aligned}$

\begin{tabular}{l|l|l|l|}
+ & 1 & 2 & 3 \\
\hline 1 & 1 & 1 & 1 \\
\hline 2 & 1 & 2 & 2 \\
\hline 3 & 1 & 2 & 3 \\
\hline
\end{tabular}

\begin{tabular}{c|c}
$a$ & $a^{\prime}$ \\
\hline 1 & 2 \\
\hline 2 & 1 \\
\hline 3 & 1 \\
\hline
\end{tabular}

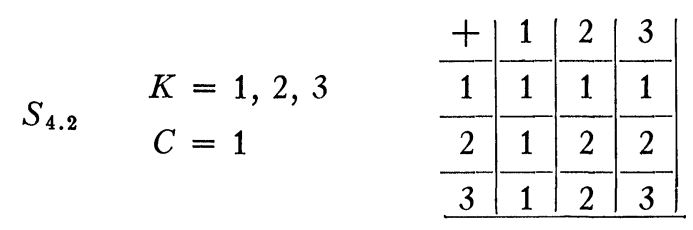

\begin{tabular}{l|l}
$a$ & $a^{\prime}$ \\
\hline 1 & 2 \\
\hline 2 & 1 \\
\hline 3 & 1 \\
\hline
\end{tabular}

Thus it follows that the four undefined ideas are all independent.

I may remark, in conclusion, that it is possible to construct a theory of the "irredundancy of undefined ideas" which is analogous to Church's "irredundancy of postulates." Undefined classes are irredundant when no one of them is a sub-class of some of the others, and similarly for relations and operations (taken in extension). Thus, in either of the examples above, $C$ is not irredundant, since it is a sub-class of $K$; though this

* $S_{3.1}$ is given by Huntington, loc. cit., to show the consistency of P1-P8. 
situation could easily be avoided by taking $C$ and $C^{\prime}$ as undefined and defining $K$ as $C+C^{\prime}$. It is doubtful, however, whether irredundancy of undefined ideas is an especially useful concept.

The University of Califororia

\section{DISTRIBUTIONS OF GREATEST VARIATES, LEAST VARIATES, AND INTERVALS OF VARIATION IN SAMPLES FROM A RECTANGULAR UNIVERSE*}

BY E. G. OLDS

1. Introduction. It is proposed to present in this paper the distributions of greatest variates, least variates, and intervals of variation, in samples of size $N$ drawn, without replacement, from the population characterized by the frequency distribution

$$
f(x)=\left\{\begin{array}{l}
1 \text { for } x=0,1,2, \cdots, b, \\
0 \text { elsewhere }
\end{array}\right.
$$

This is a finite universe of discrete variates, distributed rectangularly.

The distributions of various statistical parameters, in the case of samples from rectangular distributions, have been investigated by Rietz $\dagger$ and others, $\ddagger$ but they have been concerned with continuous distributions. The two investigations most closely related to the contents of this paper are those of $\mathrm{J}$. Neyman $\S$ and E. S. Pearson, and of P. R. Rider. I

* Presented to the Society, December 27, 1934.

$\dagger$ On a certain law of probability of Laplace, Proceedings of the International Mathematical Congress, Toronto (1924), vol. 2, pp. 795-799.

$\ddagger$ Philip Hall, The distribution of means for samples of size $N$ drawn from a population in which the variate takes values between 0 and 1 , all such values being equally probable, Biometrika, vol. 19 (1927), pp. 240-244. Allen T. Craig, On the distributions of certain statistics, American Journal of Mathematics, vol. 54 (1932), pp. 353-366.

$\S$ On the use and interpretation of certain test criteria for purposes of statistical inference, Biometrika, vol. 20A (1928), pp. 175-240.

I On the distribution of the ratio of the mean to standard deviation in small samples from non-normal universes, Biometrika, vol. 21 (1929), pp. 124-143. 